\title{
Rupture of Non-communicating Rudimentary Horn at 28 Weeks of Pregnancy in Previously Scarred Uterus: A Diagnosis in Disguise
}

\author{
Mayuri Meena, Anju Sharma \\ MS Senior Resident, Department of Obstetrics \& Gynecology SMS Medical College \& Attached Hospitals, \\ Jaipur, Rajasthan, India \\ MS Senior Professor, Department of Obstetrics \& Gynecology SMS Medical College \& Attached Hospitals, \\ Jaipur, Rajasthan, India
}

\begin{abstract}
Pregnancy in non-communicating rudimentary horn of unicornuate uterus is an uncommon form of ectopic pregnancy usually resulting in rupture during second trimester with grave consequences for the mother and fetus. We report a case of ruptured non-communicating rudimentary horn at 28 weeks of gestation in woman with previous Caesarean section in unicornuate uterus. She presented to emergency with pain abdomen since four days. Laparotomy was done and the rudimentary horn excised. Post-operative recovery was uneventful. High index of suspicion of uterine anomaly and its early diagnosis in cases of second trimester rupture is required to save this catastrophic event.
\end{abstract}

Keywords: unicornuate uterus; non-comunicating rudimentary horn; ectopic pregnancy; rupture.

\section{Introduction}

Unicornuate uterus with rudimentary horn is a rare mullerian anomaly which results from incomplete fusion of the mullerian ducts. In $83 \%$ cases of unicornuate uterus the horn is non-communicating [1]. Pregnancy in this horn is rare [2] with high incidence of obstetrical complications that include miscarriage, ectopic pregnancy, uterine rupture, preterm labour and malpresentations [3]. Risk of rupture varies from $50-90 \%$, with most ruptures occurring by the end of the second trimester because of poorly developed musculature and commonly present with abdominal pain [4]. Pre-rupture diagnosis is possible with high index of suspicion in the early pregnancy. Use of three dimensional ultrasound and magnetic resonance imaging (MRI) has been described for diagnosis but confirmation is usually surgical at laparoscopy or laparotomy [1]. Usual treatment is excision of rudimentary horn, although subtotal or total hystetrectomy may sometimes be necessary to save the life of woman. Although many cases of ectopic pregnancy in communicating horn of unicornuate uterus at an early gestation have been reported in literature but pregnancy in non-communicating rudimentary horn at 28 weeks is a rare presentation.

\section{Case report:}

A 30-year-old woman gravida 2, para 1 came to emergency with complaint amenorrhoea for seven months and pain in lower abdomen since 4 days. Pain was insidious in onset, continuous, dull aching in nature, non-radiating and non-shifting. There were no aggravating or relieving factors. There was no history of fever, vomiting, altered bowel habit or trauma. She had no ultrasound done in this pregnancy. She was not sure of her last menstrual period and denied history of dysmenorrhoea. Her previous delivery was three years back by caesarean section for cephalo-pelvic disproportion. No records of previous surgery were available. She had no significant medical or surgical history.

On examination her general condition was stable, pulse rate was 110 beats per minute, good volume and blood pressure 108/60 mm of Hg. There was mild degree of pallor. The abdomen was soft and fundal height corresponded to 28 weeks of gestation. There was no guarding or tenderness. Fetal heat sound was not localised on auscultation. Her bowel sounds were normal. On pelvic examination cervix and vagina were healthy, there was no bleeding per-vaginum. Her hemoglobin was $8 \mathrm{gm} \%$. An emergency ultrasound (USG) was carried out with the suspicion of rupture of previous caesarean scar. USG showed an empty uterus, with an echogenic structure of $81 \times 43 \mathrm{~mm}$ (probably placenta) adjacent to it. A dead fetus of 28 weeks was noted in the abdominal cavity. There was mild to moderate degree of free fluid in abdomen. Along with resuscitative measures, the patient was taken for explorative laparotomy with the provisional diagnosis of previous caesarean scar rupture. Intraoperatively about one litre of hemoperitoneum was suctioned out. A macerated fetus of $1.1 \mathrm{~kg}$ with gastroschisis was found in peritoneal cavity [Figure 1]. Placenta was located in right noncommunicating rudimentary horn of unicornuate uterus with rupture on its posterior surface [Figure 2]. Both the ovaries and 
tubes were normal. Previous caesarean scar was healthy. The rudimentary horn was excised along with the fallopian tube of same side and peritoneal lavage was done [Figure 3]. She received two units of blood transfusion and had an uneventful recovery. She was counselled for family planning and was advised for early booking and elective caesarean section in future pregnancy. She was discharged on eighth post-operative day with an advice for intravenous pyelogram six weeks later to rule out renal anomalies.

\section{Discussion}

Unicornuate uterus is a mullerian anomaly resulting from the failure in the development of one of the para-mesonephric ducts. It is sub-classified in to communicating, non-communicating, no cavity, and no horn according to the American Society of Reproductive Medicine [5].

Pregnancy in a non-communicating rudimentary horn is rare and can be explained by transperitoneal migration of the spermatozoon or the fertilized ovum and presence of a corpus luteum on the contralateral side in 8\% [6]. Most cases are diagnosed on laparotomies after uterine rupture at premature gestational age resulting in high neonatal mortality [7] and high maternal morbidity due to massive blood loss from thick and vascular uterine wall [4]. Uterine rupture associated with rudimentary horn was first reported in 1669 by Mauriceau [8].

Role of ultrasonography has been described in the diagnosis of the condition but with a low sensitivity of $26 \%$ which decreases further as the pregnancy advances [9]. Tsafrir et al outlined a set of criteria for diagnosing pregnancy in the rudimentary horn [10]. They are: (1) A pseudo pattern of asymmetrical bicornuate uterus; (2) Absent visual continuity tissue surrounding the gestation sac and the uterine cervix: (3) Presence of myometrial tissue surrounding the gestation sac. Still due to unfamiliarity of radiologist, most cases remain undiagnosed until it ruptures and presents as an emergency.

Tubal pregnancy, cornual pregnancy, and abdominal pregnancy are common sonographic and clinical misdiagnosis [4]. MRI is of proven use in diagnosis and management of such cases [11] as early diagnosis before rupture can be managed laparoscopically by immediate excision of the horn, pregnancy, and the ipsilateral fallopian tube [9].

The definitive management for both ruptured and unruptured rudimentary horn pregnancy is surgical removal of the pregnant horn [4]. We performed an emergency laparotomy and successfully excised the rudimentary horn along with ipsilateral salpingectomy. Laparoscopic excision of the horn prior to rupture has been reported successfully since last two decades [12,13]. Medical management with methotrexate during early pregnancy in the rudimentary horn has also been described [14]. Furthermore, conservative management can be advocated till the period of viability in selected cases with larger myometrial mass, if emergency surgery can be performed anytime and the patient is well-informed [5].

Approximately $36 \%$ of unicornuate uterus are associated with renal anomalies; hence it is important to assess these women and offer ureterolysis, if required, prior to the surgery [4].

In this case the condition of patient was stable except for tachycardia. This was probably due to an old rupture which can be explained by her pain abdomen since four days and a macerated fetus. Neither her previous operative records were available nor was she aware about any anomaly of her uterus. All these reasons contributed to the delayed diagnosis of rupture. Hence this case is being reported to highlight this rare but potentially lethal presentation which could be avoided by early detection and patient education. We suggest that whenever a patient presents with pain abdomen and tachycardia in second trimester, the differential diagnosis of rupture due to uterine anomaly should be kept in mind.

\section{References}

[1]. Heinanen PK. Unicornuate uterus and rudimentary horn. Fertile Steril 1997; 168: 224-30.

[2]. Nahum GG. Rudimentary uterine horn pregnancy: a case report on surviving twins delivered eight days apart. Reprod Med 1997; 42:525-32.

[3]. B. W. Rackow, A. Arici. Reproductive performance of women with müllerian anomalies. Current Opinion in Obstetrics and Gynecology 2007; 19:229-237.

[4]. G. G. Nahum. Rudimentary uterine horn pregnancy: the 20th-century worldwide experience of 588 cases. Journal of Reproductive Medicine for the Obstetrician and Gynecologist 2002; 47:151-163.

[5]. Reichman D, Laufer MR, Robinson BK. Pregnancy outcomes in unicornuate uteri: a review. Fertil Steril 2009; 91:1886-94.

[6]. Panayotidis C, Abdel-Fattah M, Leggott M: Rupture of rudimentary horn of a unicornuate uterus at 15 weeks gestation. J Obstet Gynaecol 2004; 24:323-324.

[7]. Chopra S, Keepanasseril A, Rohilla M, Bagga R, Kalra J, and JainV. Obstetric morbidity and the diagnostic dilemma in pregnancy in rudimentary horn: retrospective analysis. Archives of Gynecology and Obstetrics 2009; 280:907-910.

[8]. MauriceauF.Traite des maladies des femmes Grosses vol. 1, Compaigne des libraries, Paris, France, 1721.4.

[9]. Jayasinghe Y, Rane A, Stalewski H, Grover S. The presentation and early diagnosis of the rudimentary uterine horn. Obstet Gynecol 2005; 105:1456-67.

[10]. Tsafrir A, Rojansky N, Sela HY. Rudimentary horn pregnancy: first trimester pre-rupture sonographic diagnosis and confirmation by magnetic resonance imaging. J Ultrasound Med 2005; 24:219-223. 
[11]. Lawhon BP, Wax JR, Dufort RT. Rudimentary uterine horn pregnancy diagnosed with magnetic resonance imaging. Obstet Gynecol1998; 91: 869 .

[12]. Yan CM. Laparoscopic management of three rare types of ectopic pregnancy. Hong Kong Med J 2010; 16:132-6.

[13]. Sharma D, Usha MG, Gaikwad R, Sudha S. Laparoscopic resection of unruptured rudimentary horn pregnancy. Int J Reprod Contracept Obstet Gynecol 2013; 2:95-8.

[14]. Edelman AB, Jensen JT, Lee DM, Nichols MD. Successful medical abortion of a pregnancy within a noncommunicating rudimentary uterine horn. Am J Obstet Gynecol 2003; $189: 886-7$.

Figures

Fig. 1

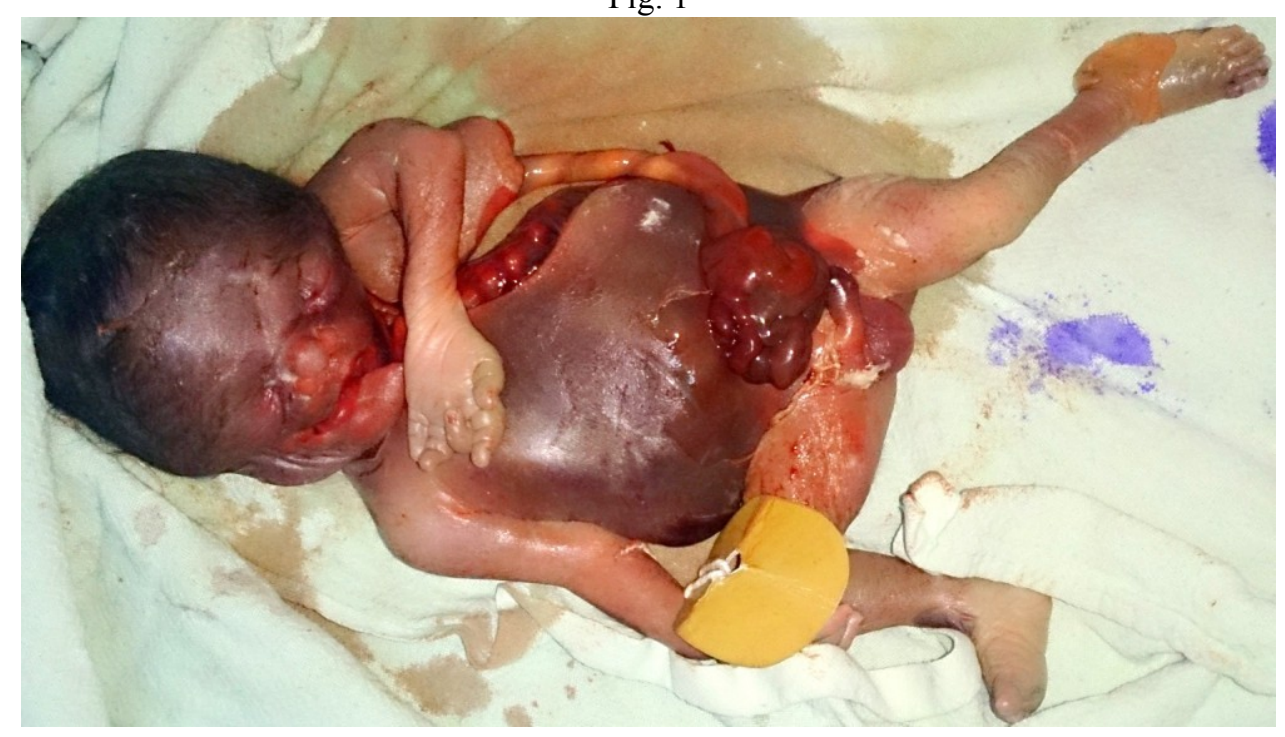

Fig 2

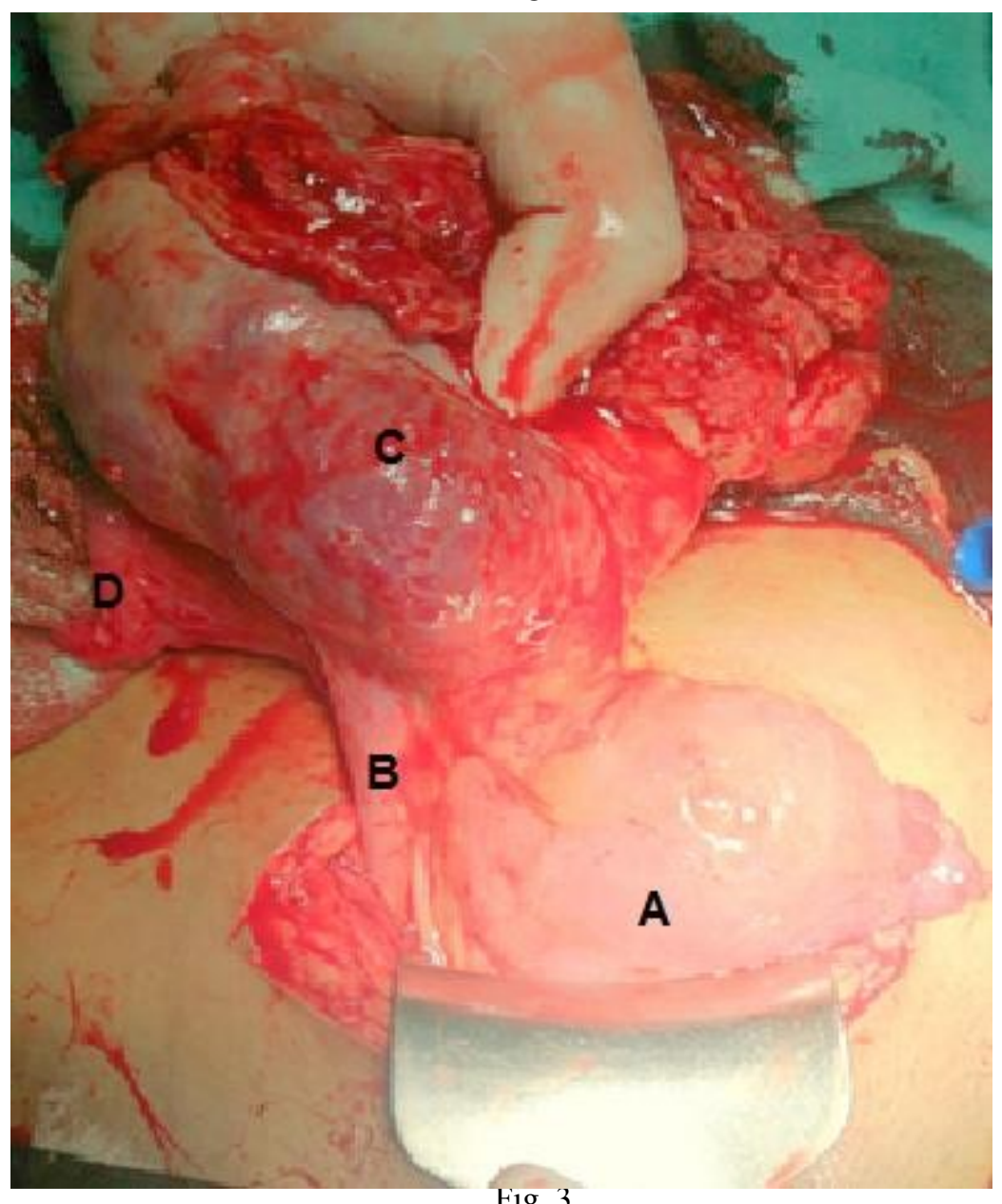




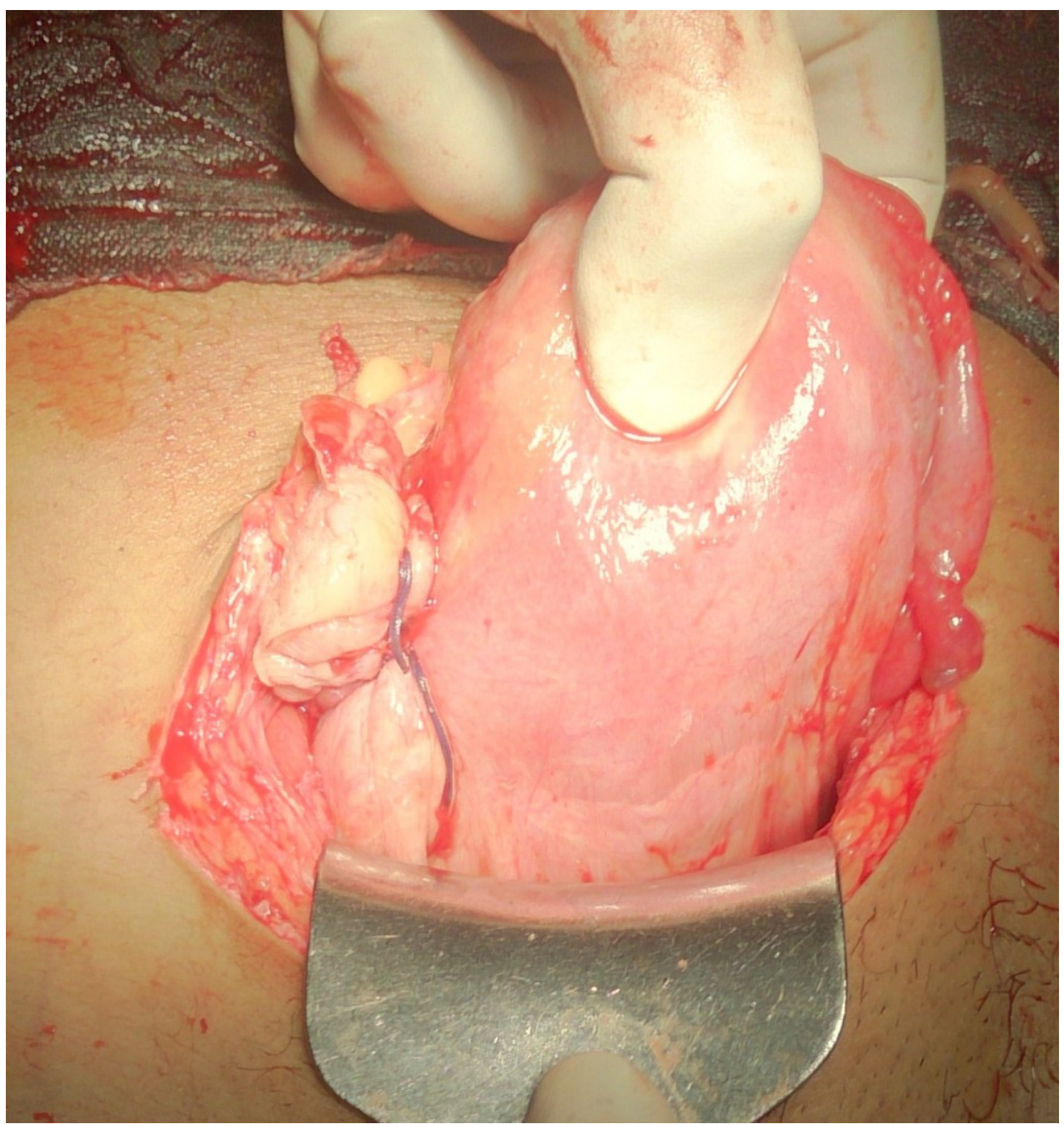

\section{Legends:}

Figure 1: Photograph showing macerated baby with gastroschisis.

Figure 2. Intra-operative photograph showing the anterior view of the unicornuate uterus (A); round ligament of right side (B); right non-communicating rudimentary horn (C) and right fallopian tube attached to the rudimentary horn $(\mathrm{D})$.

Figure 3. After excision of the rudimentary horn. 